\title{
Perioperative Management of Patients with Idiopathic Pulmonary Fibrosis Undergoing Noncardiac Surgery: A Narrative Review
}

\author{
Zyad J Carr $\mathbb{D}^{1,2}$, Luying Yan', Jose Chavez-Duarte $\mathbb{D}^{1,2}$, jill Zafar ${ }^{1,2}$, Adriana Oprea $\mathbb{D}^{1,2}$ \\ 'Yale University School of Medicine, New Haven, CT, 06520, USA; ${ }^{2}$ Department of Anesthesiology, Yale New Haven Hospital, New Haven, CT, \\ 06510, USA
}

Correspondence: Zyad J Carr, Department of Anesthesiology, Yale University School of Medicine, 333 Cedar St. TMP-3, New Haven, CT, 06520, USA, 333 Cedar St. TMP-3 Email zyad.carr@yale.edu

\begin{abstract}
Purpose: The idiopathic interstitial pneumonias (IIP) constitute a large cohort of the over 200 subtypes of interstitial lung disease (ILD). Idiopathic pulmonary fibrosis (IPF) is the most widely studied, arguably the most severe etiology of ILD and the most common IIP diagnosis. The objective of this narrative review is to outline the current evidence on optimal perioperative management of IPF. PubMed, Embase and Web of Science were analyzed for appropriate peer-reviewed references by utilizing key word search ("interstitial lung disease" OR "idiopathic pulmonary fibrosis" OR "idiopathic interstitial pneumonitis" OR "ILD” OR "IPF" AND "surgery" OR "anesthesia" OR "perioperative") within the past thirty years (1990-current). Non-English language references were excluded. A total of 205 references were curated by the authors. Eighty-seven consensus statements, clinical trials, retrospective cohort studies or case series met criteria and were incorporated into the findings of this narrative review.

Conclusion: After review, we conclude that complications, dominated by postoperative pulmonary complications, pose a significant barrier to safe perioperative care of patients with IPF. Ensuring that the preoperative IPF patient has been medically optimized is important for minimizing this risk. Initial assessment of the ARISCAT score, pulmonary function studies and cardiopulmonary exercise testing may identify IPF patients at particularly high perioperative pulmonary risk. Identifying IPF patients with 6-12month declines in $\mathrm{D}_{\mathrm{LCO}}$ of $>15 \%, \mathrm{~V}_{02 \max }<8.3 \mathrm{~mL} / \mathrm{kg} / \mathrm{min},<80 \%$ predicted value $\mathrm{FVC}$, a 50 -meter reduction in the $6 \mathrm{MWT}$ or preoperative home oxygen use may be helpful in preoperative risk stratification. Medically optimizing treatable co-morbidities should be a priority in preoperative assessment. Regional or neuraxial anesthesia should be considered an optimal technique for the avoidance of general anesthesia related complications when indicated. Acute exacerbation and postoperative pneumonia have been identified as important postsurgical complications in both thoracic and nonthoracic surgical populations.
\end{abstract}

Keywords: interstitial lung disease, lung fibrosis, perioperative, anesthesia, surgery

\section{Introduction}

ILD is comprised of a large heterogeneous group of diseases with a collective endpoint of fibrotic and inflammatory destruction of the lung parenchyma. There are over 200 causes of ILD that are generally divided into groups of identifiable (occupational, autoimmune and environmental) and unidentifiable causes (idiopathic) ${ }^{1}$ (Figure 1). Idiopathic pulmonary fibrosis (IPF) represents the most common, recognizable, and arguably, severe etiology of the subcategory of idiopathic interstitial pneumonias (IIP), comprising $50-60 \%$ of all IIP diagnoses. The purpose of this review is to analyze the perioperative risk profile of patients with IPF, identify advantageous interventions and characterize barriers to optimal care.

\section{Methodology}

PubMed, Embase and Web of Science were analyzed for appropriate citations by utilizing key word search ("interstitial lung disease" OR “idiopathic pulmonary fibrosis" OR “Idiopathic Interstitial Pneumonitis" OR "ILD” OR “IPF” AND 


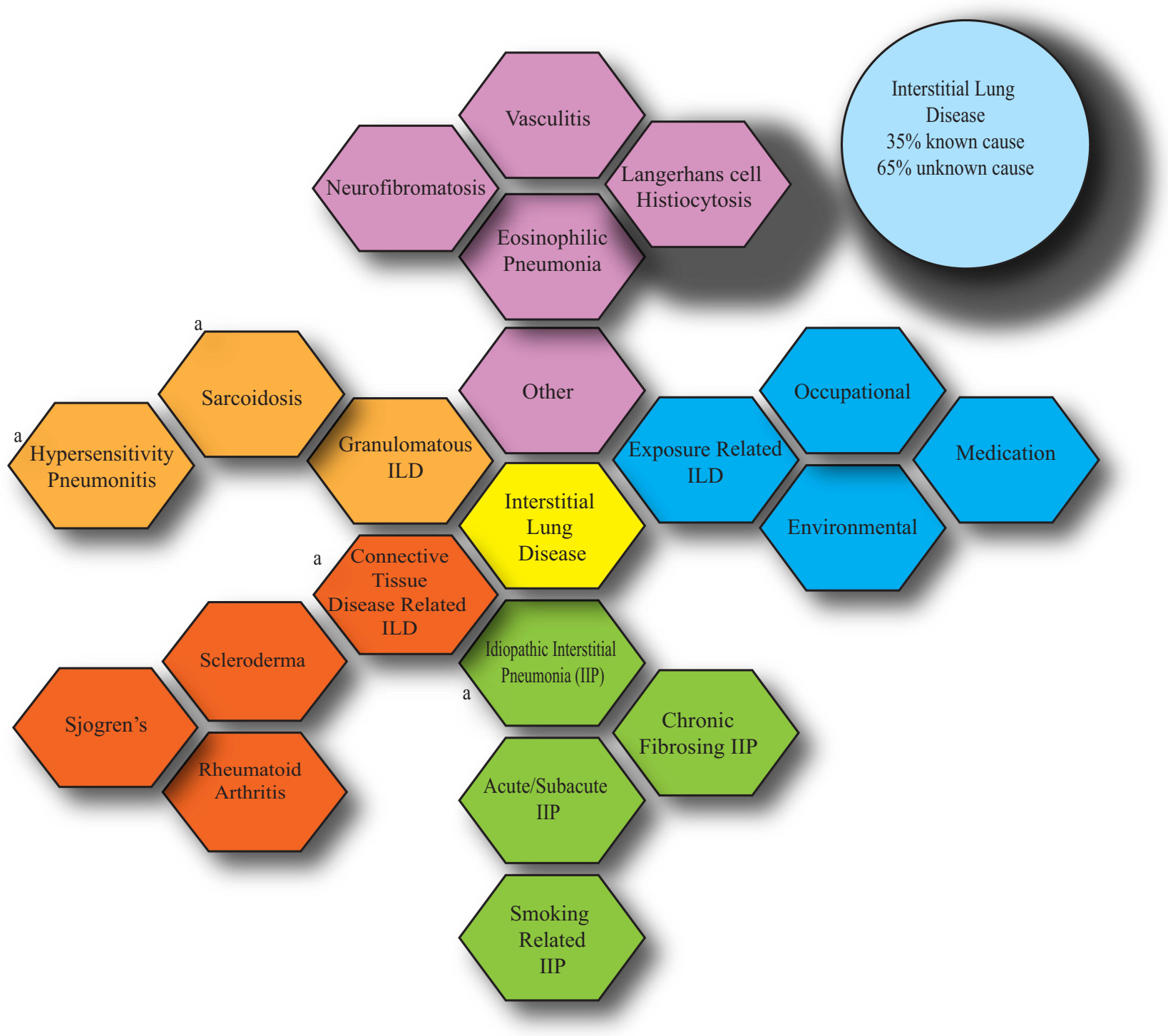

Figure I A graphical overview of interstitial lung disease. Heterogeneity of subtypes is evident, all leading to common presenting symptoms of cough, weight loss and shortness of breath. Histologically, common features of fibrotic changes are shared amongst the subtypes with subtle differences. The idiopathic interstitial pneumonias comprise the majority of identified cases. $a$ : denotes more common subtypes.

"surgery" OR "anesthesia" OR "perioperative") within the past thirty years (1990-current). Non-English language references were excluded. Two hundred and five citations were curated by the authors. Eighty-seven consensus statements, clinical trials, retrospective cohort studies or case series met criteria and were incorporated into the findings of this narrative review.

\section{Diagnostic Criteria, Clinical Findings and Epidemiology of IPF}

IPF has an incidence of 5.6/100,000 person-years in one study utilizing code-based diagnostic algorithm refinements. ${ }^{2}$ The annual cumulative prevalence is $18.2 / 100,000$ (2010) and median survival is $2-3$ years after diagnosis. ${ }^{3,4}$ IPF is the ILD diagnosis with the worst prognosis and mortality has been steadily rising, particularly in males, although this may be an artifact related to improvements in diagnosis. ${ }^{5,6}$ It occurs primarily in older adults and consists of a chronic, progressive interstitial pneumonitis with a histological and radiological pattern of usual interstitial pneumonitis (UIP). ${ }^{7}$ Patients typically present with chronic dry cough, progressive dyspnea and exhibit bibasilar crackles on physical examination. Usual investigations on presentation include chest radiography, pulmonary function testing (PFT: with a 
characteristic restrictive pattern) and high-resolution computed tomography (HRCT). The diagnosis of IPF is complex and reliant on the combination of clinical presentation pattern, progressive dyspnea, analysis of lung spirometry data from PFT studies, exclusion of other forms of ILD and the appearance of the UIP pattern on chest radiography. The UIP histopathological diagnosis has been associated with accelerated disease progression and significantly worse prognosis. ${ }^{8}$ Histopathological findings of UIP include dense fibrosis with scarring and honeycomb appearance of the lung. Additional findings of subpleural or paraseptal fibrosis distribution or patchy involvement may also be present. Disease severity in IPF is loosely classified as mild, moderate, and severe, based on the severity of respiratory limitation, and PFT studies remain the mainstay of monitoring disease progression in IPF. Decline in forced vital capacity (FVC) shows a strong correlation with decreased survival and is a validated measurement of IPF progression, with one-year risk of death twofold higher in patients with a 6 -month decline between $5 \%$ and $10 \% .{ }^{9}$ In addition, the six-minute walk test (6MWT) has been increasingly adopted as a simple assessment of cardiopulmonary, musculoskeletal, and nutritional conditioning in patients with IPF. Lower 6MWT in IPF patients is associated with increased hospitalization and mortality risk. ${ }^{10,11}$ Informal staging has been generally performed utilizing HRCT and histopathological findings. ${ }^{12}$ HRCT has increased in popularity as it provides visualization of key anatomical findings of IPF and improves prognostication and surveillance of disease progression. Visually quantified disease by HRCT has been observed to be an independent predictor of mortality in IPF (fibrosis $p=0.002$, mean lung attenuation $p=0.003$, skewness $p<0.001$ and kurtosis $p<0.001$ ) although the most commonly used measurements remain FVC and other lung spirometry variables. ${ }^{13}$ Many ILD subtypes share symptoms and histopathological features with IPF. Precise diagnosis is crucial as approximately $35 \%$ of ILD subtypes will be responsive to medical treatment. Thomeer et al found 5 -year survival rates of $91.6 \%$ for sarcoidosis, $69.7 \%$ for connective tissue disease related ILD, and $35 \%$ for IPF. ${ }^{14}$ This has led to the adoption of multidisciplinary team meetings to improve precision of IPF diagnosis leading to improved diagnostic accuracy in non-IPF experts. ${ }^{15}$

\section{Preoperative Risk Assessment and Optimization of Patients with IPF}

Treatment for IPF is generally supportive (pulmonary rehabilitation, supplemental oxygen) but has improved with the advent of antifibrotic pharmaceutical agents (pirfenidone; fibroblast inhibitor/anti-inflammatory and nintedanib; tyrosine kinase inhibitor) that have demonstrated efficacy in slowing disease progression and have shown mortality benefit. The application of nintedanib and pirfenidone for the treatment of IPF has rapidly expanded over the past decade and has been found to slow FVC decline (annual rate of decline treatment group: $-114.7 \mathrm{~mL}$ vs placebo $-239.9 \mathrm{~mL} ; \mathrm{P}<0.001$ ), reduce the risk of acute exacerbation of ILD (AE-ILD) (hospitalizations (\%) pirfenidone: 7\% vs placebo: 12\% $\mathrm{P}=0.001$ ) and improve long-term survival. ${ }^{16-18}$ Despite these promising findings, early referral for lung transplantation remains the only modality that definitively increases life expectancy for patients with end-stage IPF.

Preoperative assessment should focus on quantifying postoperative pulmonary complication (PPC) risk and identify opportunities for preoperative optimization of other, often overlooked, organ systems. This review has identified clinical evidence from both ILD and IPF clinical investigations that are useful for the extrapolation of preoperative risk in this patient population. ${ }^{19}$ In an ILD study population, notable nonmodifiable risk factors associated with in-hospital mortality after surgical lung biopsy included male gender (Odds Ratio 1.50, 95\% CI: 1.22,1.85; p $<0.001$ ), IPF classification (Odds Ratio 3.71, 95\% CI: 12.83, 4.88; $\mathrm{p}<0.001$ ) and increasing age (trend $\mathrm{p}<0.001$ ). ${ }^{20}$

PPC represents the highest risk to IPF patients and should be a primary focus of preoperative risk assessment. The most common PPC have been identified as acute respiratory worsening (ARW: new or worsened hypoxia or hypercarbia within 30 days of admission), acute exacerbation of ILD (AE-ILD: rapidly progressive clinical deterioration of ILD without obvious clinical cause), postoperative pneumonia and prolonged or occult postoperative pneumothorax. An initial assessment of the ARISCAT (Assess Respiratory Risk in Surgical Patients in Catalonia) score calculation, which assists in identifying high-risk patient for PPC, may prove useful in guiding postoperative pulmonary risk stratification. ${ }^{21}$ The ARISCAT score utilizes age, preoperative pulse oximetry, recent respiratory infection, invasiveness, urgency, and duration of the surgical procedure to calculate a risk profile for PPC, including respiratory failure. Moreover, PFT studies, cardiopulmonary exercise testing (CPET) and the 6MWT can contribute to identifying high-risk or rapidly deteriorating IPF patients presenting for surgery. For example, useful indicators of rapidly declining respiratory function include a 6-12 month decrease in the diffusion capacity for carbon monoxide $\left(\mathrm{D}_{\mathrm{LCO}}\right)$ of $>15 \%$, a $\mathrm{V}_{02} \max$ less than $8.3 \mathrm{~mL} / \mathrm{kg} / \mathrm{min}$ 
and a 6-month 50 meter reduction in the $6 \mathrm{MWT}{ }^{4}$ Another study demonstrated a correlation between preoperative home oxygen use and postoperative ARW in IPF patients, providing an easy identifier of higher-risk IPF patients (Relative Risk 2.70; 95\% CI: $1.50,4.86, \mathrm{P}<0.001) .{ }^{22}$ Furthermore, IPF patients with $<80 \% \mathrm{FVC}$ on preoperative pulmonary function testing have been found to have increased cardiopulmonary complications and worse long-term survival after lung resection. ${ }^{23,24}$

Given the high mortality of postoperative AE-ILD, preoperative identification of these patients has been an area of intensive research. Sato et al identified several factors associated with AE-ILD in IPF patients for lung resection; abnormal serum sialylated carbohydrate (KL-6) (Odds Ratio: 2.02 95\% CI: 1.19, $3.40 \mathrm{p}=0.008$ ), surgical lung resection greater than a wedge resection (Odds Ratio: $2.91,95 \%$ CI: $1.45,5.84 \mathrm{p}=0.002$ ), and FVC $<80 \%$ predicted (Odds Ratio: $0.57,95 \% \mathrm{CI}: 0.04,0.88, \mathrm{p}=0.01) .{ }^{25}$ Additionally, reviewing HRCT findings for the presence of UIP may prove valuable in identifying high-risk patients. Chida et al searched for the presence of symptomless or localized UIP in patients presenting for lung cancer resection and found that primary lung cancer resection patients with evidence of UIP on their HRCT had an $8.8 \%$ chance of acute respiratory distress syndrome (ARDS) after their procedure compared to $0.4 \%$ in patients without these findings. ${ }^{26}$

Identifying and medically optimizing potentially treatable comorbid conditions should be prioritized in the preoperative assessment of IPF patients. Survival in IPF patients is negatively impacted by coronary arterial disease, other cardiovascular disease, arteriosclerosis, and lung cancer, with median survival decreasing with increasing number of morbidities [(number of comorbidities: median survival, in months) $0: 66 ; 1-3: 48 ; 4-7: 35] .{ }^{27}$ A systematic review demonstrated high prevalence of lung cancer (3-48\%), chronic obstructive pulmonary disease [(COPD); 6-67\%], atrial fibrillation [(AF); 6-19\%], pulmonary hypertension [(PH); 3-86\%], obstructive sleep apnea [(OSA); 6-91\%] and venous thromboembolic disease [(VTE); 3-6\%]. ${ }^{28}$ IPF patients have high prevalence rates of coronary artery disease $(9.3 \%)$ and heart failure (8.2\%) compared to non-IPF patients suggesting that meticulous preoperative cardiac assessment should be performed. ${ }^{29,30} \mathrm{PH}$, defined as increased pulmonary vascular resistance of $>3$ Wood units, a mean pulmonary arterial pressure $>20 \mathrm{mmHg}$ at rest or a mean pulmonary arterial pressure of $25 \mathrm{mmHg}$ or greater obtained by right heart catheterization, is a common complication of IPF. In IPF populations, it is estimated that PH incidence ranges between $8.1 \%$ and $14.9 \%$ on diagnosis, $30-50 \%$ in advanced cases, and its presence is associated with increased morbidity and mortality. ${ }^{31,32}$ Furthermore, in IPF patients with $\mathrm{PH}$, the presence of abnormal brain natriuretic peptide (BNP) serum levels demonstrates a strong correlation with worse prognosis. ${ }^{33}$ Preoperative smoking cessation should be strongly encouraged and evidence of partial radiologic regression of interstitial lung disease has been observed in former smokers. ${ }^{34}$ Screening for OSA is recommended as it is very common in patients with IPF, with $88 \%$ of patients demonstrating an abnormal apnea-hypopnea index. ${ }^{35}$ Gastroesophageal reflux disease (GERD) is also highly prevalent in IPF patients, and there is some controversial evidence that poorly controlled GERD may contribute to disease progression and possibly higher risk for AE-ILD. ${ }^{27,36}$

Preoperative review of biomarkers may provide useful insight into the severity of lung disease in IPF patients. Abnormal preoperative C-reactive protein (CRP) level was found to be independently associated with AE-ILD after nonpulmonary surgery [OR 2.556 (95\% CI: 1.11-5.889); $\mathrm{p}=0.028] .{ }^{37}$ Serum lactate dehydrogenase (hazard ratio: $2.024, \mathrm{p}=$ 0.047 ) and serum Krebs von den Lungen-6 (KL-6) (hazard ratio: 2.909, $\mathrm{p}=0.038$ ) have been reported as accurate markers of 3-month mortality and disease activity in IPF. ${ }^{38}$ It should be noted that KL-6 serum measurements are not considered a validated component of diagnosis or management and remains investigative. Hypoalbuminemia and low serum prealbumin, a widely used indicator of underlying protein-calorie malnutrition, may be useful in identifying patients with poorer prognosis (lower survival: serum prealbumin $<0.2 \mathrm{mg} / \mathrm{L}$; serum albumin $<35 \mathrm{~g} / \mathrm{L}$ ). ${ }^{39} \mathrm{The}$ presence of chronic kidney disease (CKD) in an IPF cohort increased all-cause mortality (Hazard Ratio: 0.972, 95\% CI: 0.94, 0.99, p $=0.015$ ) with calculated estimated glomerular filtration rate (eGFR) an independent predictor of survival after age and pulmonary function data adjustment. ${ }^{40}$ In the same study, IPF patients with CKD demonstrated lower diffusing capacity of carbon monoxide (DLCO (\% predicted); $40.0 \pm 13.9$ vs $45.9 \pm 14.4, \mathrm{p}=0.04$ ) and 6-minute walking distance ((in meters) $416 \pm 129$ vs $474 \pm 84, p=0.01$ ). Preoperative risk stratification in IPF patients should attempt to identify both modifiable and nonmodifiable risk factors and are summarized in Table 1. 
Table I Preoperative Risk Factors Associated with Increased Postoperative Complications in Patients with IPF

\begin{tabular}{|c|c|c|c|}
\hline Modifiable Risk Factors & Non-Thoracic Surgery & Thoracic Surgery & References \\
\hline Low DLCO & - & • & 4 \\
\hline $\mathrm{FVC}<80 \%$ & $\infty$ & • & $23,24,60$ \\
\hline Decreased VC & $\infty$ & • & $60,62,25$ \\
\hline High BMI & $\infty$ & • & 60 \\
\hline Increasing comorbidities & $\infty$ & - & 27 \\
\hline Pulmonary hypertension & $\infty$ & • & $33,60,31,32,80$ \\
\hline Heart failure & • & • & 29,30 \\
\hline GERD (conflicting findings) & $\infty$ & • & 36,71 \\
\hline Home oxygen use & - & - & 22 \\
\hline Use of immunosuppressants & • & • & $60,62,63,25$ \\
\hline Absence of pirfenidone treatment & • & • & $17,18,64,65,66$ \\
\hline Elevated serum CRP & • & $\infty$ & 37,63 \\
\hline Elevated serum LDH & • & - & 38,63 \\
\hline Elevated serum KL-6 & $\infty$ & • & 38,62 \\
\hline Hypoalbuminemia & - & • & 39,63 \\
\hline Low BMI & • & • & 75 \\
\hline No pulmonary rehabilitation & $\infty$ & • & 87 \\
\hline \multicolumn{4}{|l|}{ Non-modifiable Risk Factors } \\
\hline Male gender & $\infty$ & • & 62,25 \\
\hline Prior history of AE-ILD & $\infty$ & • & 62,25 \\
\hline Interstitial pneumonitis on $\mathrm{CT}$ & $\infty$ & • & 26,25 \\
\hline Increasing age & • & • & 21 \\
\hline
\end{tabular}

Notes: $:$ : Yes, $\infty$ : Unknown.

Abbreviations: DLCO, diffusing capacity of lung for carbon monoxide; FVC, forced vital capacity; VC, vital capacity; BMI, body mass index; GERD, gastroesophageal reflux disease; CRP, C-reactive protein; LDH, lactate dehydrogenase; KL-6, sialylated carbohydrate antigen; AE-ILD, acute exacerbation of interstitial lung disease.

\section{Intraoperative Considerations in the Care of IPF Patients}

Several intraoperative factors have been identified as increasing the risk of PPC in IPF patients. Cardiac surgery, emergency surgery, lung resection and prolonged surgical procedures are associated with higher incidence of postoperative complications in IPF populations. Hirji et al found that ILD patients who underwent cardiac surgery had longer intensive care (ICU) stay, higher postoperative mortality and higher 30-day readmission rates compared to non-ILD populations. A comparative sub-analysis within that study found that IPF patients demonstrated higher postoperative mortality (hazard ratio 3.04, 95\% CI: 2.42, 3.80, p < 0.001), longer ICU stay ( $66 \mathrm{hr}$. vs $60 \mathrm{hrs} ., \mathrm{P}<0.001$ ) and length of stay ( 9 days vs 8 days, $\mathrm{P}<0.001)$ in comparison to non-IPF ILD patients. ${ }^{41}$ Emergency surgery $(\mathrm{OR}=23.3,95 \% \mathrm{CI}$ : $2.62,218.9, \mathrm{p}=0.005)$, lung surgery $(\mathrm{OR}=5.0,95 \% \mathrm{CI}: 1.3,18.6, \mathrm{p}=0.01)$, and longer anesthesia time $(\mathrm{OR}=1.5,95 \%$ CI: $1.1,2.2, p=0.006)$ as risk factors for PPC in ILD. ${ }^{42}$ In an IPF-specific population, increasing surgical time was an independent risk factor for postoperative ARW (Relative Risk 1.03; 95\% CI: 1.02, 1.05, P $<0.001$ ). ${ }^{22}$ Long periods of 
mechanical ventilation, likely associated with ventilator-associated lung injury, are detrimental in IPF patients. This is supported by data from a large non-surgical IPF study cohort, where higher in-hospital mortality was found with mechanical ventilation [OR 4.65 (95\% CI 3.73,5.80)], admission to the intensive care unit [OR 1.83 (95\% CI 1.52, $2.21)]$ and concurrent pneumonia [OR $1.44(95 \%$ CI $1.21,1.71)]{ }^{43}$ In that same study, an in-hospital mortality rate of 49.3\% was observed in patients receiving mechanical ventilation, suggesting that identification and benefit risk assessment of high-risk IPF patients is advised to minimize the risk for prolonged perioperative mechanical ventilation. ${ }^{44}$ The length of time of safe mechanical ventilation is unknown.

Avoidance of general endotracheal anesthesia and mechanical ventilation should be considered, when clinically indicated. This supposition is firmly supported by clinical data in patients with other types of severe lung pathology. ${ }^{45}$ When indicated, neuraxial and regional anesthesia remain an optimal technique in IPF patients and can be safely performed. ${ }^{46,47}$ In one study comparing regional anesthesia to general anesthesia for thoracoscopic lung biopsy in IPF patients, longer thoracostomy drain duration $(6.23 \pm 5.1$ days vs $3.12 \pm 1.5$ days, $\mathrm{p}=0.004)$ and longer ICU stay $(3.38$ \pm 2.1 days vs $1.09 \pm 0.7$ days, $\mathrm{p}=0.019)$ was observed in patients who received general endotracheal anesthesia. ${ }^{48} \mathrm{An}$ observational study of 30 ILD patients undergoing regional anesthesia for thoracoscopic lung biopsy found no inpatient mortality, lower major morbidity and reduced hospital stay. ${ }^{49}$

Optimal ventilator strategies for perioperative IPF patients have not been definitively characterized. In one singlecenter retrospective study, ILD patients admitted to the ICU were analyzed (67\% IPF or non IPF-idiopathic). Nonsurvivors were more likely to be postoperative, ventilated at higher airway pressures and lower tidal volumes compared to survivors. Increasing severity of acute physiology and chronic health evaluation (APACHE) III score (hazard ratio, $1.33,95 \%$ CI 1.18, 1.50) and high post end-expiratory pressure (PEEP; hazard ratio, 4.72, 95\% CI 2.06, 11.15) were independently associated with mortality risk. ${ }^{50}$ The authors concluded that patients with ILD may not benefit from an open lung approach to mechanical ventilation, an assertion that requires further clinical investigation. It is also unclear if the high oxygen fractions routinely used in perioperative settings are associated with inferior postoperative outcomes in IPF patients. Although always patient-specific, resting hypoxemia may be defined as a pulse oximetry reading of $<90 \%$ in patients with ILD. ${ }^{51}$ Oxygen therapy in IPF patients prior to AE-ILD has been observed to be independently associated with mortality (Hazard ratio 1.88; 95\% CI 1.15, 3.09, $\mathrm{p}=0.01$ ), suggestive of possible harm, although the authors were unclear to its ramifications. ${ }^{52}$ Restrictive perioperative fluid management strategies may reduce postoperative AE-ILD in IPF. Mizuno et al found a liberal intraoperative fluid management strategy (AE-ILD 8.00 \pm 4.21 $\mathrm{mL} / \mathrm{kg} / \mathrm{h}$ vs non-AE-ILD $4.99 \pm 2.86 \mathrm{~mL} / \mathrm{kg} / \mathrm{h}$ OR $1.312,95 \% \mathrm{CI}: 1.03,1.667, \mathrm{p}=0.035$ ) to be associated with higher risk of AE-ILD. ${ }^{53}$ Moreover, ILD has been identified as an independent risk factor for the development of transfusion-related acute lung injury although no definitive data on perioperative blood transfusion in IPF patients are available. ${ }^{54}$

\section{Postoperative Management: A Focus on Avoidance of Pulmonary Complications}

Postoperative management of IPF patients is appropriately focused on the prevention of PPC given its high incidence. Choi et al found an 11\% incidence of PPC [composite: in-hospital pneumonia, AE-ILD, prolonged pneumothorax ( $>7 \mathrm{~d}$ ) and others (hemothorax, pulmonary infarction, spontaneous pneumothorax)] in an all ILD population. ${ }^{42}$ Elevated postoperative oxygen demand after surgical lung biopsy in ILD populations is independently associated with major complications. ${ }^{55}$ Perioperatively, IPF patients may exhibit a wide spectrum of respiratory insult spanning from lower risk, ARW to highest risk, AE-ILD. ${ }^{56}$ It is known that AE-ILD in IPF patients may have a higher mortality compared to other forms of ILD (90-day mortality: 69\% vs 33\%; $p=0.04$ IPF vs other ILD). ${ }^{57}$ The systemic inflammatory response associated with surgery, ventilator-associated lung injury, and the IPF patient's innate propensity towards inflammation likely provide a favorable environment for the development of AE-ILD in the perioperative time period. Identification of AE-ILD poses a significant challenge in the postoperative time period, given its nonspecific and insidious constellation of clinical symptoms. Criteria for the diagnosis of AE-ILD was developed in 2012: A diagnosis of any ILD, acute worsening of less than 30 days duration and CT findings of new bilateral ground glass opacification and/or consolidation superimposed on signs of baseline interstitial lung disease. ${ }^{58}$ Hallmark histopathological findings include diffuse alveolar 
damage overlying the primary characteristic fibrotic changes of IPF. Associated risk factors include micro-aspiration of gastric contents, viral infections, secondary pulmonary hypertension, lower functional residual capacity (FVC), higher body mass index, use of immunosuppressant drugs and surgical intervention, particularly thoracic-related procedures. ${ }^{36,59}$ AE-ILD occurs in $9.3 \%$ of pulmonary resections, with a $25-43.9 \%$ mortality rate. ${ }^{60,61}$ Traditionally, AE-ILD has been thought to be predominately associated with lung resection surgery, but additional evidence has suggested that nonpulmonary surgery may present a similar risk to the IPF patient. Furuya analyzed 103 idiopathic interstitial pneumonia (IIP) patients after non-pulmonary surgery. They found an incidence of $7.8 \%$ for postoperative AE-ILD and identified the following risk factors: preoperative emergency surgery classification (Hazard Ratio, 24.713; $\mathrm{P}=0.001$ ), prednisolone use (Hazard Ratio, 1.266 P = 0.018) and elevated serum CRP (Hazard Ratio, 1.351, $\mathrm{P}=0.018$ ). ${ }^{62}$ Further supporting this observation, McDowell et al found an 5\% incidence of AE-ILD in IPF populations with no significant difference between non-thoracic and thoracic study cohorts $\left[\mathrm{N}=4\right.$ (thoracic) vs 6 (non-thoracic) $\mathrm{RR}: 1.16(\mathrm{CI}: 0.34$ to $0.99 \mathrm{Q}=4.0){ }^{22}$ Similarly, Takao et al found an incidence of $6.3 \%$ and mortality rate of $80 \%$ of AE-ILD in a cohort of all-ILD patients after non-pulmonary surgery. ${ }^{37}$

Given the encouraging results of antifibrotic treatment of IPF, some studies have tested their efficacy for the prevention of AE-ILD after thoracic surgery. In a single-arm, Phase II clinical trial, Iwata et al analyzed the efficacy of perioperative administration of pirfenidone in IPF patient for the prevention of AE-ILD after lung cancer resection. They found a significant reduction compared to known incidence with a 97.2\% AE-ILD avoidance rate (95\% CI, 85.5$99.99 \% \mathrm{p}=0.004) .{ }^{63} \mathrm{~A}$ retrospective analysis of pirfenidone use in lung cancer resection found, when patients were matched by risk score (Japanese Association for Chest Surgery risk score), significant reductions in postoperative AEILD were observed in the high-risk $(\mathrm{p}=0.048)$ but not the intermediate $(\mathrm{p}=0.106)$ or low-risk $(\mathrm{p}=0.522)$ groups. $^{64}$ Urushiyama et al found a reduced 30-day mortality (control 3.1\% vs pirfenidone 1.7\%) and postoperative severe respiratory complications (control 8.5\% vs pirfenidone $2.5 \%$; OR $0.2495 \%$ CI: $0.07-0.76, \mathrm{p}=0.015$ ) in IPF patients who received preoperative pirfenidone prior to surgery under general anesthesia. ${ }^{65}$ Furthermore, continuation of pirfenidone during the perioperative time period does not appear to significantly impact wound healing. ${ }^{66}$ Prophylactic perioperative corticosteroids have also been investigated as a method to reduce the risk of AE-ILD in IPF patients with mixed results. Administration of $125 \mathrm{mg}$ of methylprednisolone prior to pulmonary resection resulted in lower serum CRP (steroid group: $8.5 \mathrm{mg} / \mathrm{dL}$ vs control: $13.3 \mathrm{mg} / \mathrm{dL}, \mathrm{p}=0.011$ ) but did not demonstrate difference in mean hospital stay or disease-free survival. ${ }^{67}$ In a phase II clinical trial of 69 patients, low-dose methylprednisolone and sivelestat, a neutrophil elastase inhibitor, was found to be ineffective for the prevention for AE-ILD after lung cancer surgery (AEILD 5.8\% vs Expected 2.0\%). ${ }^{68}$

Additional perioperative lung insult in IPF patients may be derived from perioperative subacute or silent aspiration of gastric acid, which has been implicated in AE-ILD. Firstly, there is evidence suggesting that subacute, or silent, pulmonary aspiration may occur more frequently than previously estimated. ${ }^{18} \mathrm{~F}$-Fluorodeoxyglucose positron emission scans performed in asymptomatic non-ILD patients 48 hours after undergoing gastrointestinal endoscopy found a $3.94 \%$ incidence of abnormalities consistent with clinically silent pulmonary aspiration related lung inflammation. ${ }^{69}$ Moreover, an additional study identified abnormal amounts of gastric pepsin in the bronchoalveolar lavage of patients with AEILD. ${ }^{70}$ Though the incidence of overt perioperative pulmonary aspiration is rare, with an incidence of $1 / \sim 7000$, it is possible that the increased susceptibility to lung inflammation in IPF may result in more severe postoperative respiratory sequelae, highlighting the importance of meticulous airway and gastric content management in this surgical population. ${ }^{71}$

Rangappa et al found that, in IPF populations admitted to the ICU, pneumonia comprised $42 \%$ of admissions, $33 \%$ suspected acute exacerbation of ILD and $12.5 \%$ with unspecified postoperative respiratory failure. ${ }^{72}$ In medical populations, bacterial pneumonia was a strong risk factor for 30-day mortality in IPF patients (hazard ratio: 1.30 95\% CI: 1.04, $1.63, \mathrm{p}=0.023)$ with streptococcal pneumoniae (31.6\%), Methicillin-resistant Staphylococcus aureus (18.4\%) and Klebsiella pneumoniae (9.2\%) identified as the most common pathogens. ${ }^{73}$ This propensity for pulmonary infections is also observed in surgical populations. Choi et al found an in-hospital pneumonia rate of $3.9 \%$ in an all ILD surgical population, fourfold the risk of all surgical populations. ${ }^{42,74}$ Following noncardiac surgery in IPF patients, McDowell et al identified an $9.2 \%$ risk for 30 -day postoperative pneumonia. ${ }^{22}$ Nonsurgical, or spontaneous, pneumothorax is another common complication associated with advanced IPF, with an incidence of $11 \% .{ }^{75}$ Spontaneous pneumothorax in 
patients with IPF has a poor prognosis, is often refractory to treatment with thoracostomy tube placement and often requires surgical pleurodesis. ${ }^{76}$ In perioperative settings, persistent postoperative pneumothorax incidence may be as high as $25 \%$ after minimally invasive thoracic procedures in patients with ILD. ${ }^{77}$

Emphasizing the need to comprehensively investigate postoperative hypoxemia, the possibility of PH exacerbation should be investigated in appropriate IPF patients. In an all-cause PH populations, the risk for postoperative exacerbation of PH is highest after thoracic surgery (lung resection), thromboendarterectomy, transplantation (heart, liver, lung, kidney) and cardiac surgery (mitral valve repair/replacement, left-ventricular assist devices). ${ }^{78}$ Perioperative morbidity and mortality risk is higher in any patient with $\mathrm{PH}$, with increased incidence of overt right ventricular failure, postoperative respiratory failure, arrhythmias and heart failure. ${ }^{79}$ In IPF populations, worsening PH may present in similar fashion to ARW with exertional dyspnea and persistent postoperative oxygen requirements, complicating its diagnosis. Similarly, high prevalence rates of coronary arterial disease and congestive heart failure in IPF should mandate a high index of suspicion for postoperative myocardial infarction or decompensated heart failure as a possible etiology for postoperative respiratory insufficiency. Lastly, IPF patients are at two-fold increased risk for venous thromboembolic disease (VTE) compared to the general population, contributes to poor survival, and abnormal systemic inflammation likely plays a contributing role. ${ }^{80,81}$ Close surveillance for postoperative cardiac complications includes early detection of postoperative pulmonary hypertension, myocardial ischemia, close hemodynamic monitoring, careful maintenance of systemic perfusion, correction of electrolyte and fluid imbalances and early cardiology consultation when indicated. Given the high morbidity and mortality of IPF-related cardiopulmonary complications, postoperative hypoxemia should be aggressively investigated to exclude potentially treatable causes (Figure 2).

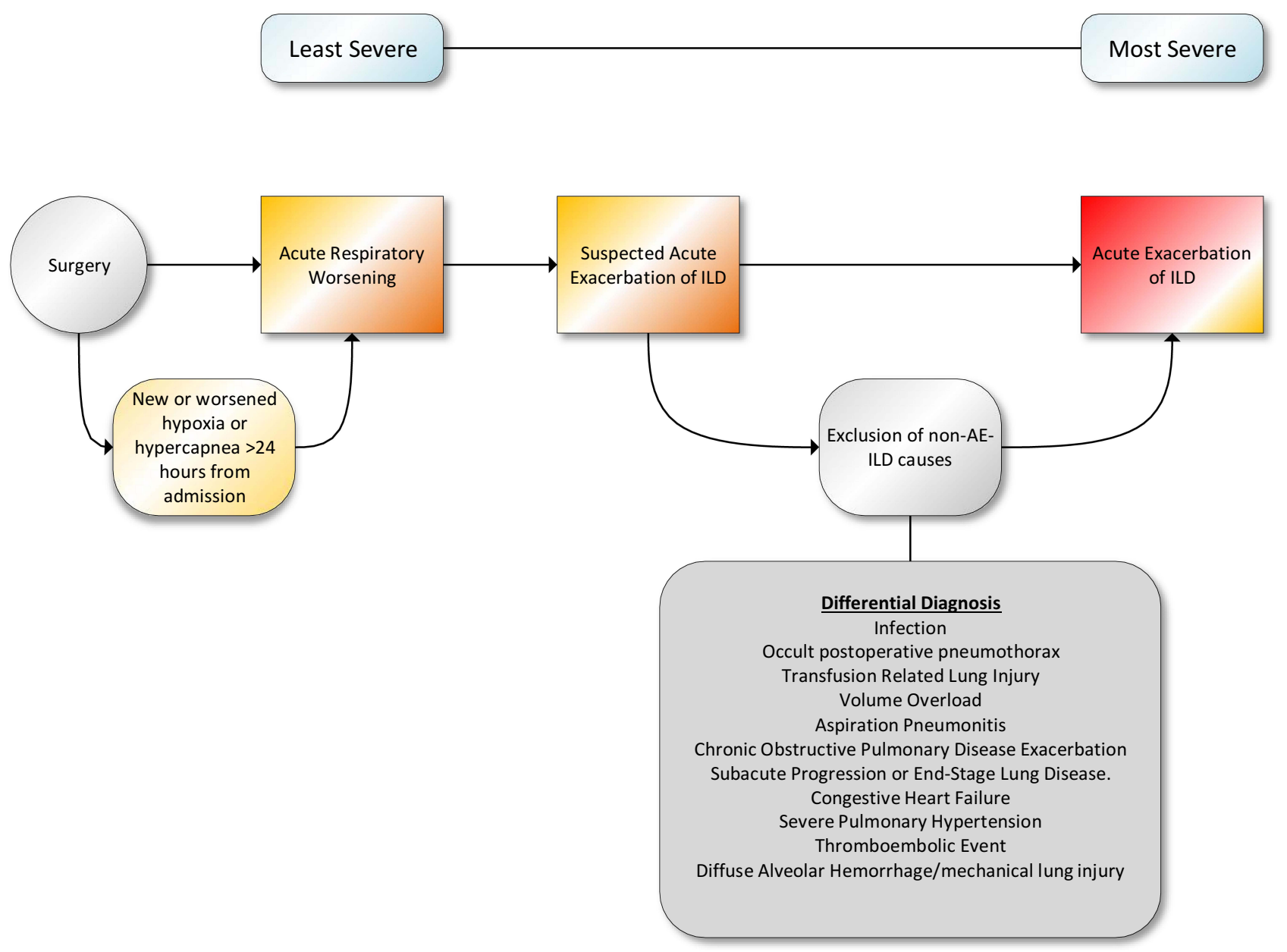

Figure 2 A methodical approach to diagnosis of postoperative hypoxemia in IPF patients is necessary to ensure that potentially treatable causes have been excluded. In addition, early pulmonary consultation may assist in rapidly diagnosing and treating AE-ILD in postoperative patients.

Abbreviations: ILD, interstitial lung disease; AE-ILD, acute exacerbation of interstitial lung disease. 
Strategies for the reduction of postoperative pulmonary complications should include low risk, but low evidence, interventions such as incentive spirometry, oral care, induced coughing, deep breathing exercises, head of bed elevation and early mobilization. ${ }^{82-84}$ Regional anesthesia and local anesthesia instillation at the surgical site may be beneficial in optimizing postoperative respiratory mechanics. There is no current evidence to support the use of prophylactic antibiotics in postoperative patients with IPF, although AE-ILD is often treated with empiric antibiotic therapy as well as high-dose corticosteroid regimens. Noninvasive positive pressure ventilation (NIPPV) may have some clinical benefit in the rescue of IPF patients with ARW. In one study, noninvasively ventilated patients spent fewer days in the ICU (in days: $11.6 \pm 4.5$ vs $24.6 \pm 13.7, \mathrm{P}=0.01$ ), suggesting that it may be useful to trial postoperative IPF patients with hypoxemia to avoid endotracheal intubation. ${ }^{85}$ The study also observed that NIPPV failure was correlated to higher serum BNP ( $>900 \mathrm{pg} / \mathrm{mL}$, OR $12.295 \%$ CI: 1.2, $\infty, \mathrm{p}=0.03$ ). Bridging the immediate postoperative extubation time period with NIPPV may provide minimally invasive respiratory support in the high-risk (residual paralysis, narcoticinduced hypercarbia, pain-related splinting) time period after surgery. Given the higher risk for VTE, early mobilization, and chemoprophylaxis should be administered as soon as possible, when clinically indicated. Although lung transplantation was not a focus of this review, significant survival benefit, reduced mechanical ventilation and reduced ICU length of stay was observed with the use of a pulmonary rehabilitation program prior to transplantation in an IPF cohort. ${ }^{86}$

\section{Conclusion}

After review, we conclude that a high incidence of postoperative complications poses a significant barrier to safe perioperative care of patients with IPF. Disease severity in IPF may be classified by severity of respiratory limitation and PFT studies are the mainstay of monitoring disease progression, particularly FVC, although the 6MWT and HRCT findings may be helpful. Ensuring that the preoperative IPF patient has been medically optimized is important for minimizing the risk of postoperative complications. Along with supportive therapies such as pulmonary rehabilitation and supplemental oxygen, the advent of antifibrotic drugs has demonstrated efficacy in slowing disease progression. Initial assessment of the ARISCAT score, PFT studies and CPET may identify IPF patients at particularly high perioperative pulmonary risk. In particular, identifying IPF patients with 6-12 month declines in $\mathrm{D}_{\mathrm{LCO}}$ of $>15 \%$, $\mathrm{V}_{02 \max }<8.3 \mathrm{~mL} / \mathrm{kg} / \mathrm{min},<80 \%$ predicted value $\mathrm{FVC}$, a 50 -meter reduction in the $6 \mathrm{MWT}$ or preoperative home oxygen use, may be helpful in preoperative risk stratification. Preoperative assessment of biomarkers can be considered as serum lactate dehydrogenase, albumin and prealbumin may identify higher risk patients. Medically optimizing treatable comorbidities should be a priority in preoperative assessment. Preoperative smoking cessation should be strongly encouraged. Identification of high-risk patients is crucial since general endotracheal anesthesia, and prolonged mechanical ventilation may induce an exaggerated inflammatory response in IPF patients, culminating in acute lung injury. Optimal ventilator strategies remain unknown in IPF patients but we can conclude that minimization of excessive oxygen fractions is reasonable. Regional or neuraxial anesthesia should be considered an optimal technique for the avoidance of general anesthesia when indicated. AE-ILD has been identified as an important postsurgical complication in both thoracic and nonthoracic surgical populations. Restrictive perioperative fluid management strategies may reduce postoperative AE-ILD. Some encouraging clinical trials have identified pirfenidone as a potential preventative regimen for acute exacerbation of ILD in the perioperative time period, but further studies are required. In addition, postoperative pulmonary infections, and persistent postsurgical pneumothorax have been identified as highly prevalent in this population and close surveillance is warranted to ensure early treatment. NIPPV may be valuable in reducing the risk of postoperative reintubation in postoperative IPF patients with hypoxemia.

Limitations of this narrative review are numerous, and we caution the reader that IPF perioperative management should be tailored to the specific needs of the patient and the procedure. The studies included in this narrative review were individually assessed for quality by the authors, but several forms of bias may have distorted their findings, include the rarity of the disorder, resulting in selection bias (numerous retrospective studies were included in this review) and unconscious confirmation bias regarding the authors' conclusions. We attempted to avoid publication bias by including relevant published negative clinical trials where available. Recommended perioperative strategies from the authors are summarized in Figure 3 but require further clinical investigation given the paucity of strong clinical evidence. In 


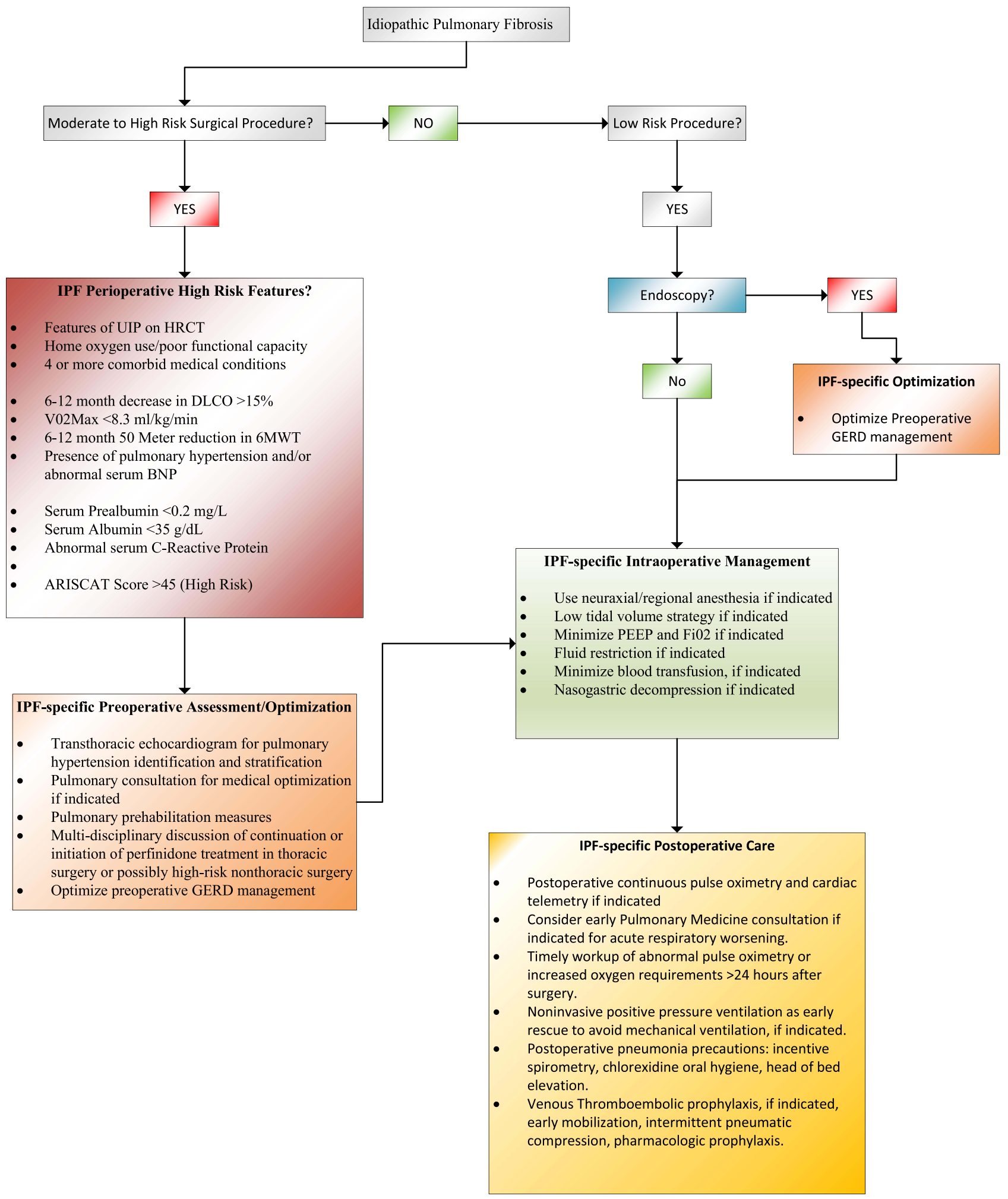

Figure 3 Optimal perioperative strategies with IPF patients focuses on identifying high-risk patients, ensuring that early risk mitigation and detection of common postoperative complications is performed in a timely fashion.

Abbreviations: IPF, idiopathic pulmonary fibrosis; UIP, usual interstitial pneumonia; HRCT, high-resolution computed tomography; DLCO, diffusing capacity for carbon monoxide; $\mathrm{V}_{\text {o2Max }}$, maximal oxygen consumption; 6MWT, six-minute walk test; BNP, NT-proB-type natriuretic peptide; PEEP, positive end-expiratory pressure; ARISCAT, Assess Respiratory Risk in Catalonia; GERD, gastroesophageal reflux disease. 
conclusion, further clinical investigations are needed to better characterize modifiable risk factors, identify lungprotective strategies, and improve perioperative outcomes in patients with IPF.

\section{Author Contributions}

All authors made a significant contribution to the work reported, whether that is in the conception, study design, execution, acquisition of data, analysis and interpretation, or in all these areas; took part in drafting, revising or critically reviewing the article; gave final approval of the version to be published; have agreed on the journal to which the article has been submitted; and agree to be accountable for all aspects of the work.

\section{Disclosure}

The authors report no conflicts of interest to disclose regarding this work.

\section{References}

1. Ryerson CJ, Collard HR. Update on the diagnosis and classification of ILD. Curr Opin Pulm Med. 2013;19(5):453-459. doi:10.1097/ MCP.0b013e328363f48d

2. Ley B, Urbania T, Husson G, et al. Code-based diagnostic algorithms for idiopathic pulmonary fibrosis. Case validation and improvement. Ann Am Thorac Soc. 2017;14(6):880-887. doi:10.1513/AnnalsATS.201610-764OC

3. Raghu G, Chen S-Y, Hou Q, Yeh W-S, Collard HR. Incidence and prevalence of idiopathic pulmonary fibrosis in US adults 18-64 years old. Eur Respir J. 2016;48(1):179-186. doi:10.1183/13993003.01653-2015

4. Ley B, Collard HR, King TE Jr. Clinical course and prediction of survival in idiopathic pulmonary fibrosis. Am J Respir Crit Care Med. 2011;183 (4):431-440. doi:10.1164/rccm.201006-0894CI

5. Mannino DM, Etzel RA, Parrish RG. Pulmonary fibrosis deaths in the United States, 1979-1991. An analysis of multiple-cause mortality data. Am J Respir Crit Care Med. 1996;153(5):1548-1552. doi:10.1164/ajrccm.153.5.8630600

6. Hutchinson JP, McKeever TM, Fogarty AW, Navaratnam V, Hubbard RB. Increasing global mortality from idiopathic pulmonary fibrosis in the twenty-first century. Ann Am Thorac Soc. 2014;11(8):1176-1185. doi:10.1513/AnnalsATS.201404-145OC

7. Raghu G, Collard HR, Egan JJ, et al. An official ATS/ERS/JRS/ALAT statement: idiopathic pulmonary fibrosis: evidence-based guidelines for diagnosis and management. Am J Respir Crit Care Med. 2011;183(6):788-824. doi:10.1164/rccm.2009-040GL

8. Bjoraker JA, Ryu JH, Edwin MK, et al. Prognostic significance of histopathologic subsets in idiopathic pulmonary fibrosis. Am J Respir Crit Care Med. 1998;157(1):199-203. doi:10.1164/ajrccm.157.1.9704130

9. du Bois RM, Weycker D, Albera C, et al. Forced vital capacity in patients with idiopathic pulmonary fibrosis: test properties and minimal clinically important difference. Am J Respir Crit Care Med. 2011;184(12):1382-1389. doi:10.1164/rccm.201105-0840OC

10. Flaherty KR, Andrei AC, Murray S, et al. Idiopathic pulmonary fibrosis: prognostic value of changes in physiology and six-minute-walk test. $A m J$ Respir Crit Care Med. 2006;174(7):803-809. doi:10.1164/rccm.200604-488OC

11. du Bois RM, Albera C, Bradford WZ, et al. 6-Minute walk distance is an independent predictor of mortality in patients with idiopathic pulmonary fibrosis. Eur Respir J. 2014;43(5):1421-1429. doi:10.1183/09031936.00131813

12. Robbie H, Daccord C, Chua F, Devaraj A. Evaluating disease severity in idiopathic pulmonary fibrosis. Eur Respir Rev. 2017;26(145):170051. doi:10.1183/16000617.0051-2017

13. Best AC, Meng J, Lynch AM, et al. Idiopathic pulmonary fibrosis: physiologic tests, quantitative CT indexes, and CT visual scores as predictors of mortality. Radiology. 2008;246(3):935-940. doi:10.1148/radiol.2463062200

14. Thomeer MJ, Vansteenkiste J, Verbeken EK, Demedts M. Interstitial lung diseases: characteristics at diagnosis and mortality risk assessment. Respir Med. 2004;98(6):567-573. doi:10.1016/j.rmed.2003.10.015

15. Walsh SLF, Maher TM, Kolb M, et al. Diagnostic accuracy of a clinical diagnosis of idiopathic pulmonary fibrosis: an international case-cohort study. Eur Respir J. 2017;50(2):1700936. doi:10.1183/13993003.00936-2017

16. Richeldi L, du Bois RM, Raghu G, et al. Efficacy and safety of nintedanib in idiopathic pulmonary fibrosis. N Engl J Med. 2014;370(22):20712082. doi:10.1056/NEJMoa1402584

17. Ley B, Swigris J, Day BM, et al. Pirfenidone reduces respiratory-related hospitalizations in idiopathic pulmonary fibrosis. Am J Respir Crit Care Med. 2017;196(6):756-761. doi:10.1164/rccm.201701-0091OC

18. Noble PW, Albera C, Bradford WZ, et al. Pirfenidone for idiopathic pulmonary fibrosis: analysis of pooled data from three multinational Phase 3 trials. Eur Respir J. 2016;47(1):243-253. doi:10.1183/13993003.00026-2015

19. Patel NM, Kulkarni T, Dilling D, Scholand MB. Preoperative evaluation of patients with interstitial lung disease. Chest. 2019;156(5):826-833. doi:10.1016/j.chest.2019.05.028

20. Hutchinson JP, Fogarty AW, McKeever TM, Hubbard RB. In-hospital mortality after surgical lung biopsy for interstitial lung disease in the United States. 2000 to 2011. Am J Respir Crit Care Med. 2016;193(10):1161-1167. doi:10.1164/rccm.201508-1632OC

21. Canet J, Gallart L, Gomar C, et al. Prediction of postoperative pulmonary complications in a population-based surgical cohort. Anesthesiology. 2010;113(6):1338-1350. doi:10.1097/ALN.0b013e3181fc6e0a

22. McDowell BJ, Karamchandani K, Lehman EB, Conboy MJ, Carr ZJ. Perioperative risk factors in patients with idiopathic pulmonary fibrosis: a historical cohort study. Can J Anaesth. 2021;68(1):81-91. doi:10.1007/s12630-020-01828-9

23. Sato T, Watanabe A, Kondo H, et al. Long-term results and predictors of survival after surgical resection of patients with lung cancer and interstitial lung diseases. J Thorac Cardiovasc Surg. 2015;149(1):64. doi:10.1016/j.jtcvs.2014.08.086

24. Sato S, Shimizu Y, Goto T, et al. Survival after repeated surgery for lung cancer with idiopathic pulmonary fibrosis: a retrospective study. $B M C$ Pulm Med. 2018;18(1):134. doi:10.1186/s12890-018-0703-8 
25. Sato T, Kondo H, Watanabe A, et al. A simple risk scoring system for predicting acute exacerbation of interstitial pneumonia after pulmonary resection in lung cancer patients. Gen Thorac Cardiovasc Surg. 2015;63(3):164-172. doi:10.1007/s11748-014-0487-6

26. Chida M, Ono S, Hoshikawa Y, Kondo T. Subclinical idiopathic pulmonary fibrosis is also a risk factor of postoperative acute respiratory distress syndrome following thoracic surgery. Eur J Cardiothorac Surg. 2008;34(4):878-881. doi:10.1016/j.ejcts.2008.07.028

27. Kreuter M, Ehlers-Tenenbaum S, Palmowski K, et al. Impact of comorbidities on mortality in patients with idiopathic pulmonary fibrosis. PLoS One. 2016;11(3):e0151425. doi:10.1371/journal.pone.0151425

28. Raghu G, Amatto VC, Behr J, Stowasser S. Comorbidities in idiopathic pulmonary fibrosis patients: a systematic literature review. Eur Respir J. 2015;46(4):1113-1130. doi:10.1183/13993003.02316-2014

29. Kato S, Kitamura H, Hayakawa K, et al. Coronary artery disease and heart failure in patients with idiopathic pulmonary fibrosis. Heart Vessels. 2021;36(8):1151-1158. doi:10.1007/s00380-021-01787-1

30. Izbicki G, Ben-Dor I, Shitrit D, et al. The prevalence of coronary artery disease in end-stage pulmonary disease: is pulmonary fibrosis a risk factor? Respir Med. 2009;103(9):1346-1349. doi:10.1016/j.rmed.2009.03.012

31. Nathan SD, Noble PW, Tuder RM. Idiopathic pulmonary fibrosis and pulmonary hypertension: connecting the dots. Am J Respir Crit Care Med. 2007;175(9):875-880. doi:10.1164/rccm.200608-1153CC

32. Seeger W, Adir Y, Barberà JA, et al. Pulmonary hypertension in chronic lung diseases. J Am Coll Cardiol. $2013 ; 62(25$ Suppl):D109-116. doi:10.1016/j.jacc.2013.10.036

33. Song JW, Song JK, Kim DS. Echocardiography and brain natriuretic peptide as prognostic indicators in idiopathic pulmonary fibrosis. Respir Med. 2009;103(2):180-186. doi:10.1016/j.rmed.2008.11.012

34. Fabre A, Treacy A, Lavelle LP, et al. Smoking-related interstitial fibrosis: evidence of radiologic regression with advancing age and smoking cessation. COPD. 2017;14(6):603-609. doi:10.1080/15412555.2017.1378631

35. Lancaster LH, Mason WR, Parnell JA, et al. Obstructive sleep apnea is common in idiopathic pulmonary fibrosis. Chest. 2009;136(3):772-778. doi: $10.1378 /$ chest.08-2776

36. Lee JS, Collard HR, Anstrom KJ, et al. Anti-acid treatment and disease progression in idiopathic pulmonary fibrosis: an analysis of data from three randomised controlled trials. Lancet Respir Med. 2013;1(5):369-376. doi:10.1016/S2213-2600(13)70105-X

37. Takao S, Masuda T, Yamaguchi K, et al. High preoperative C-reactive protein level is a risk factor for acute exacerbation of interstitial lung disease after non-pulmonary surgery. Medicine. 2019;98(5):e14296. doi:10.1097/MD.0000000000014296

38. Kishaba T, Tamaki H, Shimaoka Y, Fukuyama H, Yamashiro S. Staging of acute exacerbation in patients with idiopathic pulmonary fibrosis. Lung. 2014;192(1):141-149. doi:10.1007/s00408-013-9530-0

39. Li B, Zhang X, Xu G, et al. Serum prealbumin is a prognostic indicator in idiopathic pulmonary fibrosis. Clin Respir J. 2019;13(8):493-498. doi: $10.1111 /$ crj.13050

40. Ikezoe K, Handa T, Tanizawa K, et al. Chronic kidney disease predicts survival in patients with idiopathic pulmonary fibrosis. Respiration. 2017;94 (4):346-354. doi:10.1159/000478787

41. Hirji SA, Ramirez-Del Val F, Ejiofor JI, et al. Significance of interstitial lung disease on outcomes following cardiac surgery. Am J Cardiol. 2019;124(7):1133-1139. doi:10.1016/j.amjcard.2019.06.031

42. Choi SM, Lee J, Park YS, et al. Postoperative pulmonary complications after surgery in patients with interstitial lung disease. Respiration. 2014;87 (4):287-293. doi:10.1159/000357046

43. Durheim MT, Judy J, Bender S, et al. In-hospital mortality in patients with idiopathic pulmonary fibrosis: a US cohort study. Lung. 2019;197 (6):699-707. doi:10.1007/s00408-019-00270-z

44. Rush B, Wiskar K, Berger L, Griesdale D. The use of mechanical ventilation in patients with idiopathic pulmonary fibrosis in the United States: a nationwide retrospective cohort analysis. Respir Med. 2016;111:72-76. doi:10.1016/j.rmed.2015.12.005

45. Hausman MS Jr., Jewell ES, Engoren M. Regional versus general anesthesia in surgical patients with chronic obstructive pulmonary disease: Does avoiding general anesthesia reduce the risk of postoperative complications? Anesth Analg. 2015;120(6):1405-1412. doi:10.1213/ ANE.0000000000000574

46. Honma K, Tango Y, Isomoto H. Perioperative management of severe interstitial pneumonia for rectal surgery: a case report. Kurume Med J. 2007;54(3-4):85-88. doi:10.2739/kurumemedj.54.85

47. Rodgers A, Walker N, Schug S, et al. Reduction of postoperative mortality and morbidity with epidural or spinal anaesthesia: results from overview of randomised trials. Br Med J. 2000;321(7275):1493-1497. doi:10.1136/bmj.321.7275.1493

48. Hajjar WM, Al-Nassar SA, Al-Sugair GS, et al. Evaluation of safety and efficacy of regional anesthesia compared with general anesthesia in thoracoscopic lung biopsy procedure on patient with idiopathic pulmonary fibrosis. Saudi J Anaesth. 2018;12(1):46-51. doi:10.4103/sja. SJA 26517

49. Pompeo E, Rogliani P, Cristino B, Schillaci O, Novelli G, Saltini C. Awake thoracoscopic biopsy of interstitial lung disease. Ann Thorac Surg. 2013;95(2):445-452. doi:10.1016/j.athoracsur.2012.10.043

50. Fernandez-Perez ER, Yilmaz M, Jenad H, et al. Ventilator settings and outcome of respiratory failure in chronic interstitial lung disease. Chest. 2008;133(5):1113-1119. doi:10.1378/chest.07-1481

51. Singh S, Sharma BB, Bairwa M, et al. Management of interstitial lung diseases: a consensus statement of the Indian Chest Society (ICS) and National College of Chest Physicians (NCCP). Lung India. 2020;37(4):359-378. doi:10.4103/lungindia.lungindia_275_20

52. Kamiya H, Panlaqui OM. Systematic review and meta-analysis of prognostic factors of acute exacerbation of idiopathic pulmonary fibrosis. $B M J$ Open. 2020;10(6):e035420. doi:10.1136/bmjopen-2019-035420

53. Mizuno Y, Iwata H, Shirahashi K, et al. The importance of intraoperative fluid balance for the prevention of postoperative acute exacerbation of idiopathic pulmonary fibrosis after pulmonary resection for primary lung cancer. Eur J Cardiothorac Surg. 2012;41(6):e161-165. doi:10.1093/ejcts/ ezs 147

54. Yokoyama A, Sakamoto Y, Jo T, et al. Pulmonary disease as a risk factor for transfusion-related acute lung injury. ERJ Open Res. 2021;7(3):000392021. doi:10.1183/23120541.00039-2021

55. Yi E, Lee JH, Lee JH, Chung JH, Lee Y, Lee S. Perioperative risk factors in surgical lung biopsy for the diagnosis of interstitial lung disease: a single-centre experience. ANZ J Surg. 2020;91(3):291-297. doi:10.1111/ans.16112 
56. Moua T, Westerly BD, Dulohery MM, Daniels CE, Ryu JH, Lim KG. Patients with fibrotic interstitial lung disease hospitalized for acute respiratory worsening: a large cohort analysis. Chest. 2016;149(5):1205-1214. doi:10.1016/j.chest.2015.12.026

57. Tachikawa R, Tomii K, Ueda H, et al. Clinical features and outcome of acute exacerbation of interstitial pneumonia: collagen vascular diseasesrelated versus idiopathic. Respiration. 2012;83(1):20-27. doi:10.1159/000329893

58. Collard HR, Ryerson CJ, Corte TJ, et al. Acute exacerbation of idiopathic pulmonary fibrosis. An international working group report. Am J Respir Crit Care Med. 2016;194(3):265-275. doi:10.1164/rccm.201604-0801CI

59. Qiu M, Chen Y, Ye Q. Risk factors for acute exacerbation of idiopathic pulmonary fibrosis: a systematic review and meta-analysis. Clin Respir J. 2018;12(3):1084-1092. doi:10.1111/crj.12631

60. Alam N, Park BJ, Wilton A, et al. Incidence and risk factors for lung injury after lung cancer resection. Ann Thorac Surg. 2007;84(4):1085-1091. doi:10.1016/j.athoracsur.2007.05.053

61. Sato T, Teramukai S, Kondo H, et al. Impact and predictors of acute exacerbation of interstitial lung diseases after pulmonary resection for lung cancer. Journal of Thoracic and Cardiovascular Surgery. 2014;147(5):1604. doi:10.1016/j.jtcvs.2013.09.050

62. Furuya K, Sakamoto S, Takai Y, Sato N, Matsumoto K, Homma S. Acute exacerbation of idiopathic interstitial pneumonia after nonpulmonary surgery under general anesthesia: a retrospective study. Sarcoidosis Vasc Diffuse Lung Dis. 2017;34(2):156-164.

63. Iwata T, Yoshino I, Yoshida S, et al. A phase II trial evaluating the efficacy and safety of perioperative pirfenidone for prevention of acute exacerbation of idiopathic pulmonary fibrosis in lung cancer patients undergoing pulmonary resection: West Japan Oncology Group $6711 \mathrm{~L}$ (PEOPLE Study). Respir Res. 2016;17(1):90. doi:10.1186/s12931-016-0398-4

64. Kanayama M, Mori M, Matsumiya H, et al. Perioperative pirfenidone treatment for lung cancer patients with idiopathic pulmonary fibrosis. Surg Today. 2020;50(5):469-474. doi:10.1007/s00595-019-01923-5

65. Urushiyama H, Jo T, Hasegawa W, et al. Preoperative use of pirfenidone and reduced risk of postoperative severe respiratory complications in patients with idiopathic pulmonary fibrosis: propensity score-matched analysis using a nationwide database in Japan. Respirology. 2021;26(6):590596. doi:10.1111/resp. 14044

66. Mortensen A, Cherrier L, Walia R. Effect of pirfenidone on wound healing in lung transplant patients. Multidiscip Respir Med. $2018 ; 13: 13$. doi:10.1186/s40248-018-0124-9

67. Yano T, Koga T. [Prophylactic administration of steroid for interstitial pneumonia after pulmonary resection for lung cancer]. Kyobu Geka. 2005;58 (1):37-40. Japanese

68. Ito H, Nakayama H, Yokose T, et al. A prophylaxis study of acute exacerbation of interstitial pneumonia after lung cancer surgery. Jpn J Clin Oncol. 2020;50(2):198-205. doi:10.1093/jjco/hyz164

69. Hsieh TC, Wu YC, Ding HJ, et al. Clinically unrecognized pulmonary aspiration during gastrointestinal endoscopy with sedation: a potential pitfall interfering the performance of 18F-FDG PET for cancer screening. Eur J Radiol. 2011;80(3):e510-515. doi:10.1016/j.ejrad.2010.10.030

70. Lee JS, Song JW, Wolters PJ, et al. Bronchoalveolar lavage pepsin in acute exacerbation of idiopathic pulmonary fibrosis. Eur Respir J. 2012;39 (2):352-358. doi:10.1183/09031936.00050911

71. Sakai T, Planinsic RM, Quinlan JJ, Handley LJ, Kim TY, Hilmi IA. The incidence and outcome of perioperative pulmonary aspiration in a University hospital: a 4-year retrospective analysis. Anesth Analg. 2006;103(4):941-947. doi:10.1213/01.ane.0000237296.57941.e7

72. Rangappa P, Moran JL. Outcomes of patients admitted to the intensive care unit with idiopathic pulmonary fibrosis. Crit Care Resusc. 2009;11 (2):102-109.

73. Oda K, Yatera K, Fujino Y, et al. Respiratory comorbidities and risk of mortality in hospitalized patients with idiopathic pulmonary fibrosis. Respir Investig. 2018;56(1):64-71. doi:10.1016/j.resinv.2017.09.006

74. Chughtai M, Gwam CU, Khlopas A, et al. The incidence of postoperative pneumonia in various surgical subspecialties: a dual database analysis. Surg Technol Int. 2017;30:45-51.

75. Franquet T, Giménez A, Torrubia S, Sabaté JM, Rodriguez-Arias JM. Spontaneous pneumothorax and pneumomediastinum in IPF. Eur Radiol. 2000;10(1):108-113. doi:10.1007/s003300050014

76. Nishimoto K, Fujisawa T, Yoshimura K, et al. The prognostic significance of pneumothorax in patients with idiopathic pulmonary fibrosis. Respirology. 2018;23(5):519-525. doi:10.1111/resp.13219

77. Luo Q, Han Q, Chen X, Xie J, Wu L, Chen R. The diagnosis efficacy and safety of video-assisted thoracoscopy surgery (VATS) in undefined interstitial lung diseases: a retrospective study. J Thorac Dis. 2013;5(3):283-288. doi:10.3978/j.issn.2072-1439.2013.04.12

78. Hill NS, Roberts KR, Preston IR. Postoperative pulmonary hypertension: etiology and treatment of a dangerous complication. Respir Care. 2009;54 (7):958-968. doi:10.4187/002013209793800439

79. McGlothlin D, Ivascu N, Heerdt PM. Anesthesia and pulmonary hypertension. Prog Cardiovasc Dis. 2012;55(2):199-217. doi:10.1016/j. pcad.2012.08.002

80. Boonpheng B, Ungprasert P. Risk of venous thromboembolism in patients with idiopathic pulmonary fibrosis: a systematic review and metaanalysis. Sarcoidosis Vasc Diffuse Lung Dis. 2018;35(2):109-114. doi:10.36141/svdld.v35i2.6213

81. Sprunger DB, Olson AL, Huie TJ, et al. Pulmonary fibrosis is associated with an elevated risk of thromboembolic disease. Eur Respir J. 2012;39 (1):125-132. doi:10.1183/09031936.00041411

82. Eltorai AEM, Szabo AL, Antoci V Jr., et al. Clinical effectiveness of incentive spirometry for the prevention of postoperative pulmonary complications. Respir Care. 2018;63(3):347-352. doi:10.4187/respcare.05679

83. Caparelli ML, Shikhman A, Jalal A, Oppelt S, Ogg C, Allamaneni S. Prevention of postoperative pneumonia in noncardiac surgical patients: a prospective study using the national surgical quality improvement program database. Am Surg. 2019;85(1):8-14. doi:10.1177/ 000313481908500104

84. Ståhl A, Westerdahl E. Postoperative physical therapy to prevent hospital-acquired pneumonia in patients over 80 years undergoing hip fracture surgery-A quasi-experimental study. Clin Interv Aging. 2020;15:1821-1829. doi:10.2147/CIA.S257127

85. Vianello A, Arcaro G, Battistella L, et al. Noninvasive ventilation in the event of acute respiratory failure in patients with idiopathic pulmonary fibrosis. J Crit Care. 2014;29(4):562-567. doi:10.1016/j.jcrc.2014.03.019

86. Florian J, Watte G, Teixeira PJZ, et al. Pulmonary rehabilitation improves survival in patients with idiopathic pulmonary fibrosis undergoing lung transplantation. Sci Rep. 2019;9(1):9347. doi:10.1038/s41598-019-45828-2 


\section{Publish your work in this journal}

The International Journal of General Medicine is an international, peer-reviewed open-access journal that focuses on general and internal medicine, pathogenesis, epidemiology, diagnosis, monitoring and treatment protocols. The journal is characterized by the rapid reporting of reviews, original research and clinical studies across all disease areas. The manuscript management system is completely online and includes a very quick and fair peer-review system, which is all easy to use. Visit http://www.dovepress.com/testimonials.php to read real quotes from published authors.

Submit your manuscript here: https://www.dovepress.com/international-journal-of-general-medicine-journal 